\title{
Light polarization by biological nanocoatings
}

Mikhail Kryuchkov', Vladimir Savitsky², Bodo D. Wilts ${ }^{3}$, Elmer Gray, Vladimir L. Katanaev $v^{1,5, *}$

${ }^{1}$ Department of Cell Physiology and Metabolism, Faculty of Medicine, University of Geneva, CMU, Rue Michel Servet 1, CH-1211, Geneva, Switzerland.

2Zoological Museum of the Lomonosov Moscow State University, Bol'shaya Nikitskaya str. 2, Moscow, 125009, Russian Federation.

${ }^{3}$ Adolphe Merkle Institute, University of Fribourg, Chemin des Verdiers 4, $\mathrm{CH}-1700$, Fribourg, Switzerland. 4Department of Entomology, University of Georgia, Biological Sciences Building 413, GA 30602 Athens, USA.

${ }^{5}$ School of Biomedicine, Far Eastern Federal University, Sukhanova Street 8, Vladivostok, 690922, Russian Federation.

*Vladimir.Katanaev@unige.ch

\section{Supporting Information}



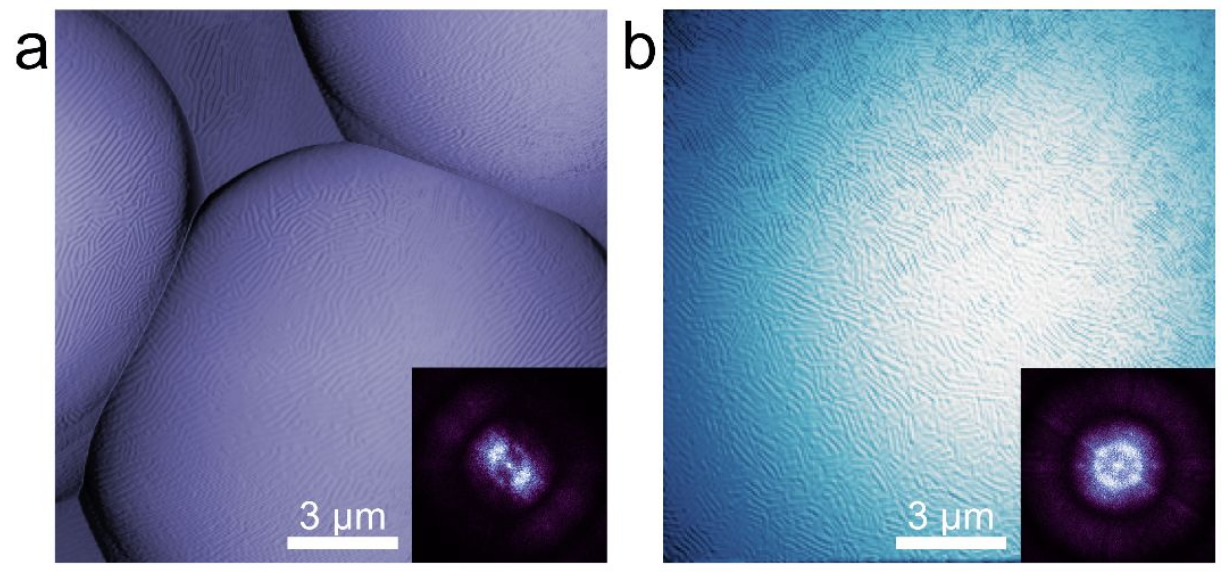

Figure S1. a,b) Atomic force microscopy of $S$. vittatum male's ventral (a) and dorsal (b) parts of eye $(15 \times 15 \mu \mathrm{m})$. Fourier transform spectra of the AFM images are shown as inserts. Note that parallel ridges retain their unidirectionality not only within the lens of one ommatidium, but also across neighboring ommatidia.
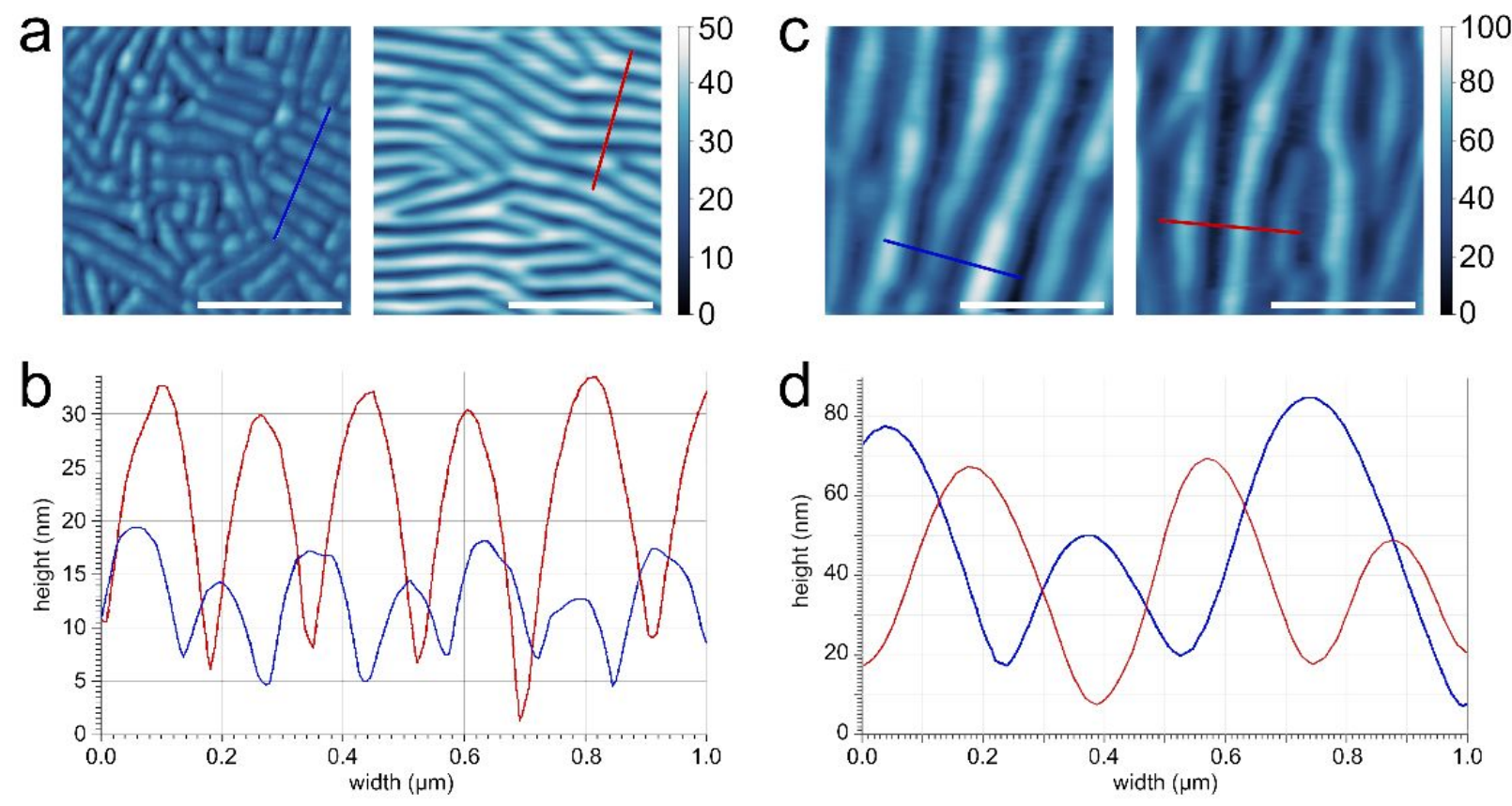

Figure S2. a) Atomic force microscopy of male $S$. vittatum dorsal (left) and ventral (right) parts of eye reveal clear difference between the nanostructural coatings of the two eye parts. b) Cross-sectional profiles ( $1 \mu \mathrm{m}$-long) of dorsal (in blue) and ventral (in red) cornea. The locations of the cross-sectioned zones are indicated by the blue and red bars on the AFM scans above. c) Representative AFM scans of $A$. lateralis lantern cuticle. d) Cross-sectional profiles ( $1 \mu \mathrm{m}$-long) of the lantern cuticle. Bars on the AFM scans above 
indicates the locations of the cross-sectioned zones. The color scale shown next to the images indicates the surface height (in nm); for all AFM images scale bars are $1 \mu \mathrm{m}$.
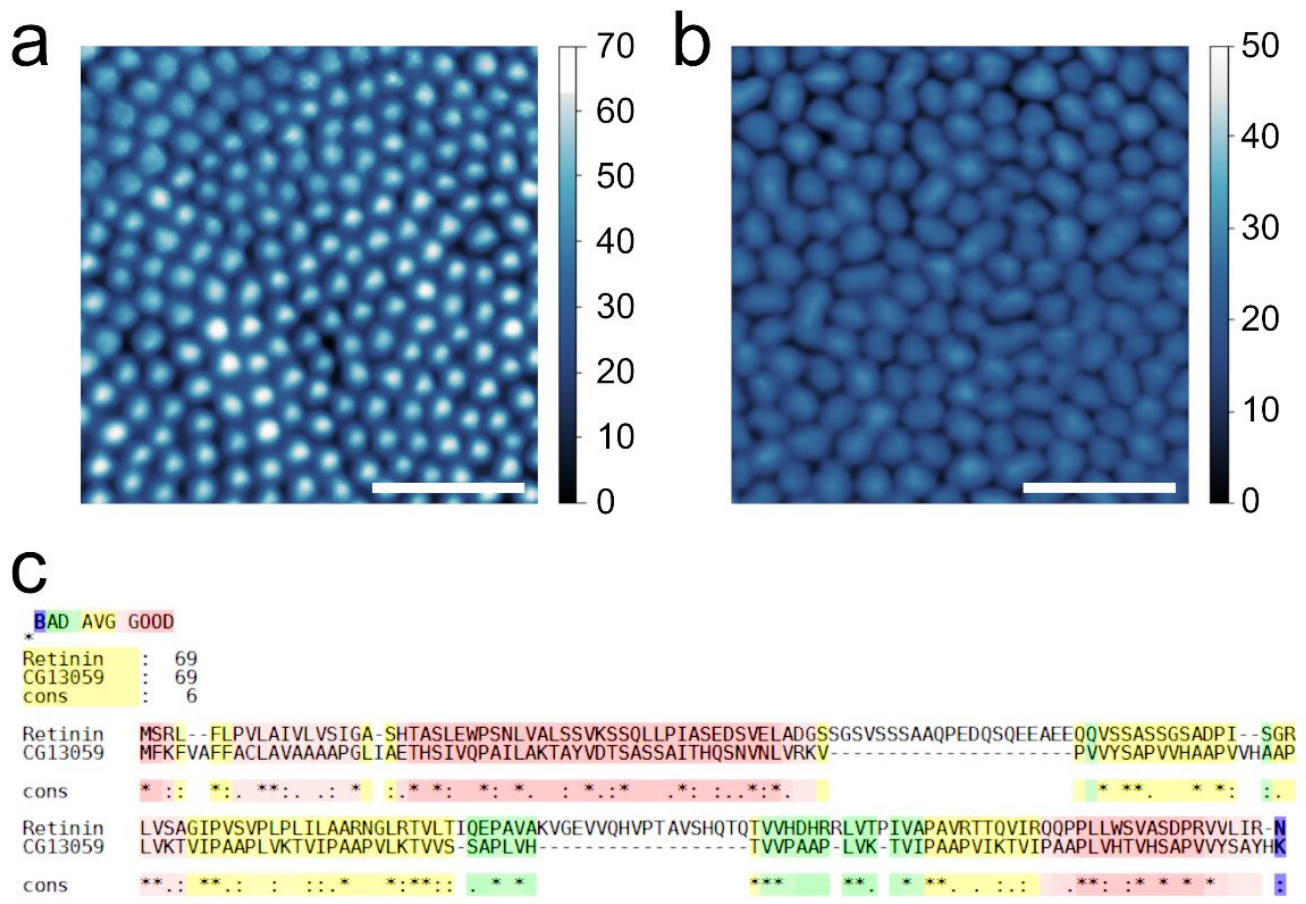

Figure S3. a,b) Atomic force microscopy images of cornea of wild type D. melanogaster (a) and upon eyespecific overexpression of $C G 13059$ (b). A clear difference in height, width and form of protrusions could be seen. The color scale shown next to the images indicates the surface height (in nm); scale bars are $1 \mu \mathrm{m}$. c) T-Coffee-based alignment of the amino acid sequences of proteins Retinin and CG13059 from $D$. melanogaster.
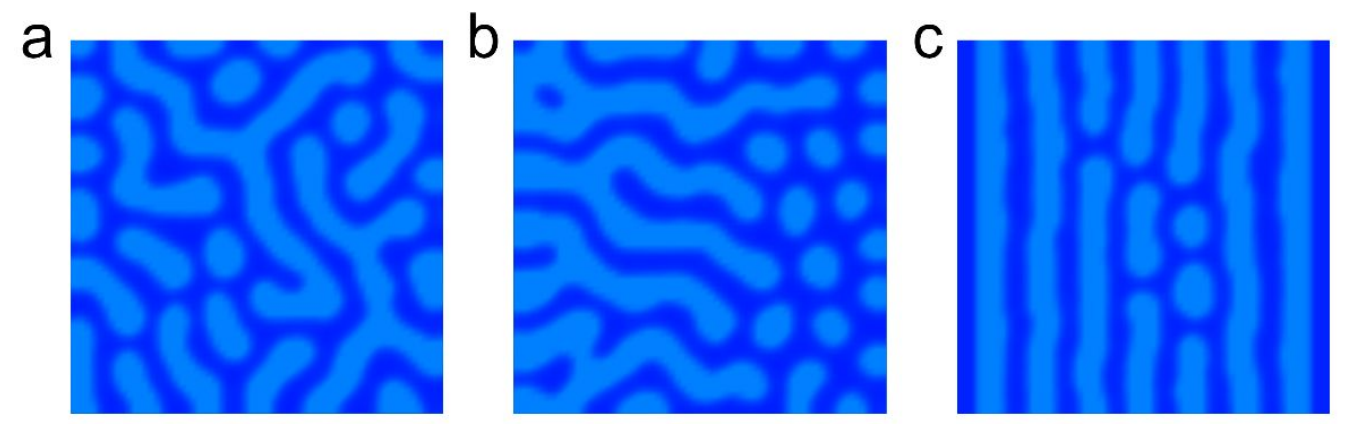

Figure S4. Results of the Turing modeling ${ }^{20}$ predict that, compare to normal unordered pattern formation (a), gradient of inhibitor's and activator's interactions' parameters is able to form parallel structures (b), as well as presence of "scratches" with absence of inhibitor (c). For the simulation, the software 'Ready' (a cross-platform implementation of various reaction-diffusion 
systems (https://github.com/GollyGang/ready)) was used with following parameters: $\mathrm{da}=0.03$, $\mathrm{Da}=0.02, \mathrm{sa}=2, \mathrm{aa}=0.07, \mathrm{ia}=-0.08446$ (b: -0.08 to $-0.08446 \mathrm{OX}$ gradient), $\mathrm{db}=0.08, \mathrm{Db}=0.45, \mathrm{sb}=1$, $a b=0.09476$ (b: 0.09 to 0.09476 OX gradient), $i b=-0.07$. For the panel $\mathbf{c}$ vertical rectangles aligned with borders with concentration of inhibitor, equal to zero were used.

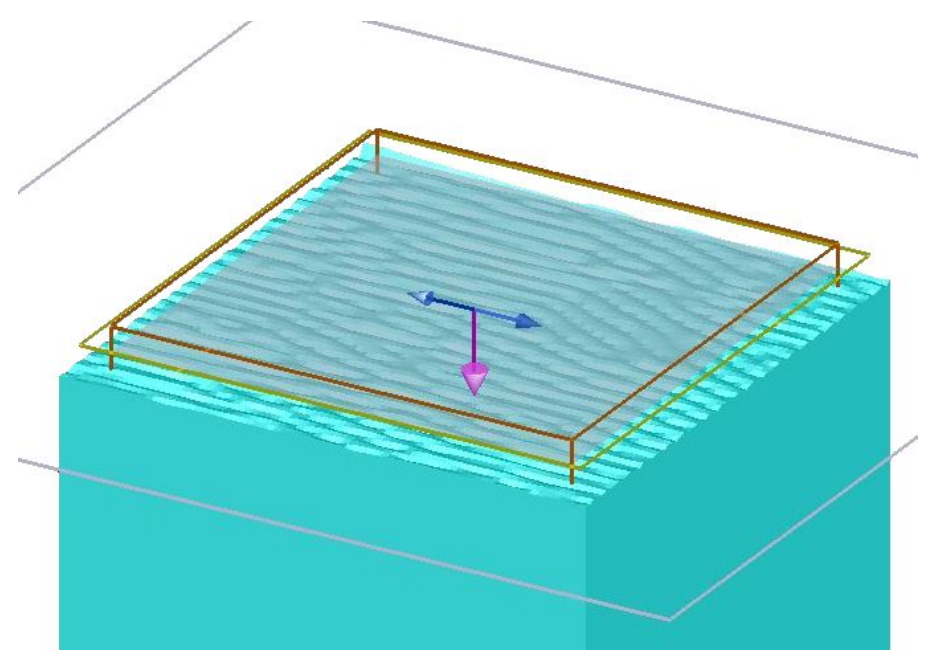

Figure S5. The view of simulation volume used for FDTD modelling. Pink arrow indicate the direction of incident light, blue arrows - the linear polarization at the exact moment $\left(\mathrm{t}_{0}\right)$. 